\title{
Weighted Distances Based on Neighbourhood Sequences in Non-standard Three-Dimensional Grids
}

\author{
Robin Strand \\ Centre for Image Analysis, Uppsala University, \\ Box 337, SE-75105 Uppsala, Sweden \\ robin@cb.uu.se
}

\begin{abstract}
By combining weighted distances and distances based on neighbourhood sequences, a new family of distance functions with potentially low rotational dependency is obtained. The basic theory for these distance functions, including functional form of the distance between two points, is presented for the face-centered cubic grid and the body-centered cubic grid. By minimizing an error function, the optimal combination of weights and neighbourhood sequence is derived.
\end{abstract}

\section{Introduction}

When using non-standard grids such as the face-centered cubic (fcc) grid and the body-centered cubic (bcc) grid for 3D images, less samples are needed to obtain the same representation/reconstruction quality compared to the cubic grid [1]. This is one reason for the increasing interest in using these grids in e.g. image acquisition [1], image processing [234], and image visualization [5].

Measuring distances is of great importance in many applications. Because of its low rotational dependency, the Euclidean distance is often used as distance function. There are, however, applications where other distance functions are better suited. For example, when minimal cost-paths are computed, a distance function defined as the shortest path between any two points is better suited, see e.g. [6], where the constrained distance transform is computed using the Euclidean distance resulting in a complex algorithm. The corresponding algorithm using a path-based approach is simple, fast, and easy to generalize to higher dimensions [7. Examples of path-based distances are weighted distances, where weights define the cost (distance) between neighbouring grid points 8,32 , and distances based on neighbourhood sequences (ns-distances), where the cost is fixed but the adjacency relation is allowed to vary along the path 94 . These path-based distance functions are generalizations of the well-known city-block and chessboard distance function defined for the square grid in [10. The rotational dependency of weighted distances and ns-distances can be minimized by optimizing the weights 8332 and neighbourhood sequences 94 , respectively.

In this paper, weighted distances and ns-distances for the fcc and bcc grids, presented in [23] and 4] respectively, are combined by defining the distance 
as the shortest path between two grid points using both weights for the local distance between neighbouring grid points and allowing the adjacency relation to vary along the path. Functional forms are given of the distance functions for grid points in the fcc and bcc grids. Moreover, the rotational dependency is minimized by finding the weights and neighbourhood sequence that minimize an error function. The results are compared with results for weighted distances and ns-distances, which are both special cases of the proposed distance function.

\section{Basic Notions}

The following definitions of the fcc and bcc grids are used:

$$
\begin{aligned}
& \mathbb{F}=\{(x, y, z): x, y, z \in \mathbb{Z} \text { and } x+y+z \equiv 0 \quad(\bmod 2)\} \\
& \mathbb{B}=\{(x, y, z): x, y, z \in \mathbb{Z} \text { and } x \equiv y \equiv z \quad(\bmod 2)\}
\end{aligned}
$$

When the result is valid for both $\mathbb{F}$ and $\mathbb{B}$, the notation $\mathbb{G}$ is used. Two grid points $\mathbf{p}_{1}=\left(x_{1}, y_{1}, z_{1}\right), \mathbf{p}_{2}=\left(x_{2}, y_{2}, z_{2}\right) \in \mathbb{G}$ are $\rho$-neighbours, $1 \leq \rho \leq 2$, if

1. $\left|x_{1}-x_{2}\right|+\left|y_{1}-y_{2}\right|+\left|z_{1}-z_{2}\right| \leq 3$ and

2. $\max \left\{\left|x_{1}-x_{2}\right|,\left|y_{1}-y_{2}\right|,\left|z_{1}-z_{2}\right|\right\} \leq \rho$

The points $\mathbf{p}_{1}, \mathbf{p}_{2}$ are adjacent if $\mathbf{p}_{1}$ and $\mathbf{p}_{2}$ are $\rho$-neighbours for some $\rho$. The $\rho$-neighbours which are not $(\rho-1)$-neighbours are called strict $\rho$-neighbours. The neighbourhood relations are visualized in Figure 1 by showing the Voronoi regions (the voxels) corresponding to some adjacent grid points.
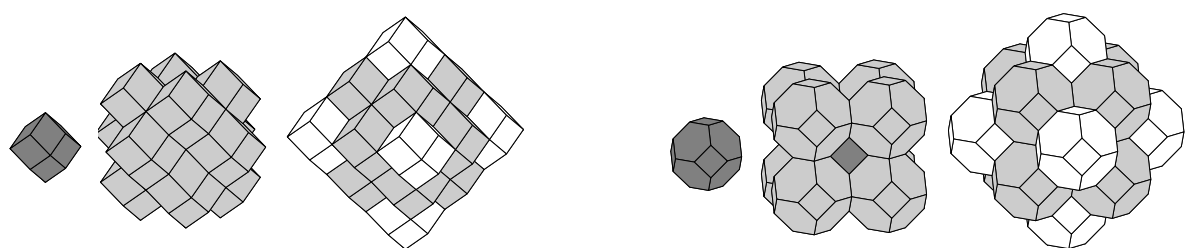

Fig. 1. The grid points corresponding to the dark and the light grey voxels are 1neighbours. The grid points corresponding to the dark grey and white voxels are (strict) 2-neighbours. Left: fcc, right: bcc.

A ns $B$ is a sequence $B=(b(i))_{i=1}^{\infty}$, where each $b(i)$ denotes a neighbourhood relation in $\mathbb{G}$. If $B$ is periodic, i.e., if for some fixed strictly positive $l \in \mathbb{Z}_{+}$, $b(i)=b(i+l)$ is valid for all $i \in \mathbb{Z}_{+}$, then we write $B=(b(1), b(2), \ldots, b(l))$. A path, denoted $\mathcal{P}$, in a grid is a sequence $\mathbf{p}_{0}, \mathbf{p}_{1}, \ldots, \mathbf{p}_{n}$ of adjacent grid points. A path is a $B$-path of length $n$ if, for all $i \in\{1,2, \ldots, n\}, \mathbf{p}_{i-1}$ and $\mathbf{p}_{i}$ are $b(i)$-neighbours. The notation 1 - and (strict) 2 -steps will be used for a step to a 1-neighbour and step to a (strict) 2-neighbour, respectively. The number of 1 -steps and strict 2 -steps in a given path $\mathcal{P}$ is denoted $\mathbf{1}_{\mathcal{P}}$ and $\mathbf{2}_{\mathcal{P}}$, respectively. 
Definition 1. Given the ns $B$, the ns-distance $d\left(\mathbf{p}_{0}, \mathbf{p}_{n} ; B\right)$ between the points $\mathbf{p}_{0}$ and $\mathbf{p}_{n}$ is the length of (one of) the shortest B-path $(s)$ between the points.

Let the real numbers $\alpha$ and $\beta$ (the weights) and a path $\mathcal{P}$ of length $n$, where exactly $l(l \leq n)$ adjacent grid points in the path are strict 2-neighbours, be given. The length of the $(\alpha, \beta)$-weighted $B$-path $\mathcal{P}$ is $(n-l) \alpha+l \beta$. The $B$-path $\mathcal{P}$ between the points $\mathbf{p}_{0}$ and $\mathbf{p}_{n}$ is a shortest $(\alpha, \beta)$-weighted $B$-path between the points $\mathbf{p}_{0}$ and $\mathbf{p}_{n}$ if no other $(\alpha, \beta)$-weighted $B$-path between the points is shorter than the length of the $(\alpha, \beta)$-weighted $B$-path $\mathcal{P}$.

Definition 2. Given the ns $B$ and the weights $\alpha, \beta$, the weighted ns-distance $d_{\alpha, \beta}\left(\mathbf{p}_{0}, \mathbf{p}_{n} ; B\right)$ is the length of (one of) the shortest $(\alpha, \beta)$-weighted B-path $(s)$ between the points.

The following notation is used:

$$
\begin{aligned}
& \mathbf{1}_{B}^{k}=|\{i: b(i)=1,1 \leq i \leq k\}| \text { and } \\
& \mathbf{2}_{B}^{k}=|\{i: b(i)=2,1 \leq i \leq k\}| .
\end{aligned}
$$

\section{Distance Function in Discrete Space}

We now state a functional form of the distance between two grid points $(0,0,0)$ and $(x, y, z)$, where $x \geq y \geq z \geq 0$. We remark that by translation-invariance and symmetry, the distance between any two grid points is given by the formula below. The formulas in Lemma 1 are proved (as Theorem 2 and 5) in [4].

Lemma 1. Let the ns $B$ and the point $\mathbf{p}=(x, y, z) \in \mathbb{G}$, where $x \geq y \geq z \geq 0$ be given. The ns-distance between $\mathbf{0}$ and $\mathbf{p}$ is given by

$$
\begin{aligned}
& d(\mathbf{0}, \mathbf{p} ; B)=\min \left\{k \in \mathbb{N}: k \geq \max \left(\frac{x+y+z}{2}, x-\mathbf{2}_{B}^{k}\right)\right\} \text { for } \mathbf{p} \in \mathbb{F} \\
& d(\mathbf{0}, \mathbf{p} ; B)=\min \left\{k \in \mathbb{N}: k \geq \max \left(\frac{x+y}{2}, x-\mathbf{2}_{B}^{k}\right)\right\} \quad \text { for } \mathbf{p} \in \mathbb{B}
\end{aligned}
$$

Given a shortest $B$-path $\mathcal{P}$ of length $\hat{k}$ (obtained by the formula in Lemma 11), Lemma 2 and 3 give the number of 1 -steps and 2 -steps in $\mathcal{P}$, i.e. $\mathbf{1}_{\mathcal{P}}$ and $\mathbf{2}_{\mathcal{P}}$, respectively.

Lemma 2. Let the point $(x, y, z) \in \mathbb{F}$, where $x \geq y \geq z \geq 0$, and the value of $\hat{k}=\min \left\{k: k \geq \max \left(\frac{x+y+z}{2}, x-\mathbf{2}_{B}^{k}\right)\right\}$ be given. If only the steps $(1,1,0)$, $(1,-1,0),(0,1,1),(1,0,1)$, and $(2,0,0)$ are used for a shortest $B$-path between $\mathbf{0}$ and $(x, y, z)$, then

$$
\mathbf{1}_{\mathcal{P}}=\left\{\begin{array}{cl}
\hat{k} & \text { if } x \leq y+z \\
2 \hat{k}-x & \text { otherwise }
\end{array}\right.
$$

and

$$
\mathbf{2}_{\mathcal{P}}=\left\{\begin{array}{cl}
0 & \text { if } x \leq y+z \\
x-\hat{k} & \text { otherwise }
\end{array}\right.
$$


Proof. First of all, in the proof of Theorem 2 in 4 it is shown that there is a shortest $B$-path with only the steps $(1,1,0),(1,-1,0),(0,1,1),(1,0,1)$, and $(2,0,0)$ when $x \geq y \geq z \geq 0$.

For the case $x \leq y+z$, the length of the shortest $B$-path is independent of $B$ and there is a shortest $B$-path consisting of only 1 -steps, see Theorem 2 in [4. Hence, the number of 1 -steps is $\hat{k}$ and the number of 2 -steps is 0 .

In the proof of Theorem 2 in [4, it is shown that only the steps $(2,0,0)$, $(1,1,0),(1,-1,0)$, and $(1,0,1)$ are needed to find a shortest $B$-path between $(0,0,0)$ and $(x, y, z) \in \mathbb{F}$, where $x \geq y \geq z \geq 0$ and $x>y+z$. Thus, $(x, y, z)=$ $(2,0,0) \mathbf{a}_{1}+(1,1,0) \mathbf{a}_{2}+(1,-1,0) \mathbf{a}_{3}+(1,0,1) \mathbf{a}_{4}$ for some $\mathbf{a}_{1}, \mathbf{a}_{2}, \mathbf{a}_{3}, \mathbf{a}_{4} \in \mathbb{N}$. Obviously, the length $\hat{k}$ of the path is $\mathbf{a}_{1}+\mathbf{a}_{2}+\mathbf{a}_{3}+\mathbf{a}_{4}, \mathbf{1}_{\mathcal{P}}=\mathbf{a}_{2}+\mathbf{a}_{3}+\mathbf{a}_{4}$, and $\mathbf{2}_{\mathcal{P}}=\mathbf{a}_{1}$. We get:

$$
\begin{aligned}
& \hat{k}=\mathbf{a}_{1}+\mathbf{a}_{2}+\mathbf{a}_{3}+\mathbf{a}_{4} \\
& x=2 \mathbf{a}_{1}+\mathbf{a}_{2}+\mathbf{a}_{3}+\mathbf{a}_{4} .
\end{aligned}
$$

Thus, $\mathbf{1}_{\mathcal{P}}=\mathbf{a}_{2}+\mathbf{a}_{3}+\mathbf{a}_{4}=2 \hat{k}-x$ and $\mathbf{2}_{\mathcal{P}}=\mathbf{a}_{1}=x-\hat{k}$.

Lemma 3. Let the point $(x, y, z) \in \mathbb{B}$, where $x \geq y \geq z \geq 0$, and the value of $\hat{k}=\min \left\{k: k \geq \max \left(\frac{x+y}{2}, x-\mathbf{2}_{B}^{k}\right)\right\}$ be given. If only the steps $(1,1,1)$, $(1,1,-1),(1,-1,-1)$, and $(2,0,0)$ are used for a shortest $B$-path between $\mathbf{0}$ and $(x, y, z)$, then

$$
\mathbf{1}_{\mathcal{P}}=2 \hat{k}-x
$$

and

$$
\mathbf{2}_{\mathcal{P}}=x-\hat{k}
$$

Proof. In the proof of Theorem 5 in 4, it is shown that only the steps $(2,0,0)$, $(1,1,1),(1,1,-1)$, and $(1,-1,-1)$ are needed to find a shortest $B$-path between $(0,0,0)$ and $(x, y, z) \in \mathbb{B}$, where $x \geq y \geq z \geq 0$. Thus, $(x, y, z)=(2,0,0) \mathbf{a}_{1}+$ $(1,1,1) \mathbf{a}_{2}+(1,1,-1) \mathbf{a}_{3}+(1,-1-1) \mathbf{a}_{4}$ for some $\mathbf{a}_{1}, \mathbf{a}_{2}, \mathbf{a}_{3}, \mathbf{a}_{4} \in \mathbb{N}$. Obviously, $\mathbf{1}_{\mathcal{P}}=\mathbf{a}_{2}+\mathbf{a}_{3}+\mathbf{a}_{4}$ and $\mathbf{2}_{\mathcal{P}}=\mathbf{a}_{1}$. Using the same technique as in the proof of Lemma 2, the result follows.

Lemma 4. Let the $n s B$ and the point $(x, y, z) \in \mathbb{G}$, where $x \geq y \geq z \geq 0$ be given. If $\mathcal{P}$ is a shortest B-path between $\mathbf{0}$ and $(x, y, z)$ consisting of only the steps

$$
\begin{array}{r}
(2,0,0),(1,1,0),(1,-1,0),(0,1,1), \text { and }(1,0,1) \quad(f c c) \\
(2,0,0),(1,1,1),(1,1,-1), \text { and }(1,-1,-1) \quad(b c c)
\end{array}
$$

such that the weights $\alpha, \beta \in \mathbb{R}$ are such that $0<\alpha \leq \beta \leq 2 \alpha$, then $\mathcal{P}$ is also a shortest $(\alpha, \beta)$-weighted $B$-path between $\mathbf{0}$ and $(x, y, z)$.

Proof. From [4, we know that a shortest $B$-path is obtained by using these steps when $x \geq y \geq z \geq 0$, cf. Lemma 2 and 3 . 
Let $n$ be the length of $\mathcal{P}$ and let $l$ be the number of 2 -steps in $\mathcal{P}$. The length of $\mathcal{P}$ can then be written $n=(n-l) 1+l 1$. The length of the $(\alpha, \beta)$-weighted $B$-path is $(n-l) \alpha+l \beta$. Assume that the shortest $(\alpha, \beta)$-weighted $B$-path $\mathcal{P}^{\prime}$ is a shorter $(\alpha, \beta)$-weighted $B$-path between $\mathbf{0}$ and $(x, y, z)$ than $\mathcal{P}$. Let the length of $\mathcal{P}^{\prime}\left((1,1)\right.$-weighted) be $n^{\prime}\left(n^{\prime} \geq n\right.$ since $\mathcal{P}$ is a shortest $B$-path) and the number of 2 -steps be $l^{\prime}$. The length of the $(\alpha, \beta)$-weighted $B$-path $\mathcal{P}^{\prime}$ is $\left(n^{\prime}-l^{\prime}\right) \alpha+l^{\prime} \beta$. By assumption,

$$
\left(n^{\prime}-l^{\prime}\right) \alpha+l^{\prime} \beta<(n-l) \alpha+l \beta .
$$

We get the following cases:

i $l^{\prime}<l$

Since $\mathcal{P}^{\prime}$ is a path between $\mathbf{0}$ and $(x, y, z)$ and the only 2 -step that is used is $(2,0,0)$, there are at least $2\left(l-l^{\prime}\right)$ more 1 -steps in $\mathcal{P}^{\prime}$ compared to $\mathcal{P}$. We get $\left(n^{\prime}-l^{\prime}\right) \geq(n-l)+2\left(l-l^{\prime}\right) \Rightarrow\left(n^{\prime}-l^{\prime}\right)-(n-l) \geq$ $2\left(l-l^{\prime}\right) \Rightarrow\left(\left(n^{\prime}-l^{\prime}\right)-(n-l)\right) \alpha \geq 2\left(l-l^{\prime}\right) \alpha$. By the assumption (3) , we have $\left(\left(n^{\prime}-l^{\prime}\right)-(n-l)\right) \alpha<\left(l-l^{\prime}\right) \beta$. Thus, $2\left(l-l^{\prime}\right) \alpha \leq\left(\left(n^{\prime}-l^{\prime}\right)-(n-l)\right) \alpha<$ $\left(l-l^{\prime}\right) \beta \Rightarrow 2 \alpha<\beta$, which is a contradiction.

ii $l^{\prime}>l$

$$
\begin{aligned}
\left(n^{\prime}-l^{\prime}\right) \alpha+l^{\prime} \beta & <(n-l) \alpha+l \beta \quad \text { by }(3) \\
\left(n-l^{\prime}\right) \alpha+l^{\prime} \beta & <(n-l) \alpha+l \beta \text { since } n^{\prime} \geq n \\
\left(l^{\prime}-l\right) \beta & <\left(l^{\prime}-l\right) \alpha \\
\beta & <\alpha \quad \text { since } l^{\prime}>l,
\end{aligned}
$$

which is a contradiction.

This proves that the assumption (3) implies $l^{\prime}=l$. Rewriting (3) with $l^{\prime}=l$ gives $n^{\prime}<n$, which contradicts the fact that $\mathcal{P}$ is a shortest $B$-path (i.e. that $\left.n^{\prime} \geq n\right)$. Therefore (3) is false, so

$$
\left(n^{\prime}-l^{\prime}\right) \alpha+l^{\prime} \beta \geq(n-l) \alpha+l \beta .
$$

It follows that $\mathcal{P}$ is a shortest $(\alpha, \beta)$-weighted $B$-path.

The following theorems (Theorem 1 and 2) are obtained by summing up the results from Lemma 14 4. Given a shortest $B$-path consisting of as many 1 -steps as possible between 0 and $(x, y, z) \in \mathbb{G}$ with $x \geq y \geq z \geq 0$, if $0<\alpha \leq \beta \leq 2 \alpha$, we know by Lemma 4 that the path is also a shortest $(\alpha, \beta)$-weighted $B$-path. Using Lemma 2 and 3, where the number of 1-steps and 2-steps in the path are given, the formulas in Lemma 1 can be rewritten such that the length of the path is given by summing the number of 1-steps and 2-steps. By multiplying these numbers with the corresponding weights, we get the following functional forms of the weighted distance based on neighbourhood sequences between two points in the fcc and bcc grids.

Theorem 1. Let the ns $B$, the weights $\alpha$, $\beta$ s.t. $0<\alpha \leq \beta \leq 2 \alpha$, and the point $(x, y, z) \in \mathbb{F}$, where $x \geq y \geq z \geq 0$, be given. The weighted ns-distance between $\mathbf{0}$ and $(x, y, z)$ is given by 


$$
\begin{aligned}
d_{\alpha, \beta}(\mathbf{0},(x, y, z) ; B) & =\left\{\begin{array}{cc}
k \cdot \alpha & \text { if } x \leq y+z \\
(2 k-x) \cdot \alpha+(x-k) \cdot \beta & \text { otherwise, }
\end{array}\right. \\
\text { where } k & =\min _{k}: k \geq \max \left(\frac{x+y+z}{2}, x-\mathbf{2}_{B}^{k}\right) .
\end{aligned}
$$

Theorem 2. Let the ns $B$, the weights $\alpha$, $\beta$ s.t. $0<\alpha \leq \beta \leq 2 \alpha$, and the point $(x, y, z) \in \mathbb{B}$, where $x \geq y \geq z \geq 0$, be given. The weighted ns-distance between $\mathbf{0}$ and $(x, y, z)$ is given by

$$
\begin{aligned}
d_{\alpha, \beta}(\mathbf{0},(x, y, z) ; B) & =(2 k-x) \cdot \alpha+(x-k) \cdot \beta \\
\text { where } k & =\min _{k}: k \geq \max \left(\frac{x+y}{2}, x-\mathbf{2}_{B}^{k}\right) .
\end{aligned}
$$

To illustrate the discrete distance functions, balls of radius 20 in the fcc grid with $\alpha=1, \beta=1.4862$, and $B=(1,2)$ and the bcc grid with $\alpha=1, \beta=1.2199$, and $B=(1,2)$ are shown in Figure 2

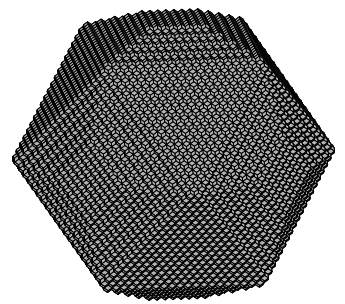

(a)

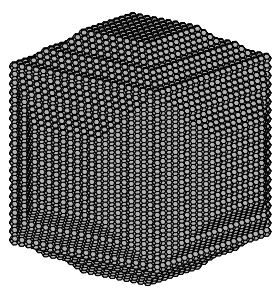

(b)

Fig. 2. Balls of radius 20 in the fcc grid with $\alpha=1, \beta=1.4862$, and $B=(1,2)$ (a) and the bcc grid with $\alpha=1, \beta=1.2199$, and $B=(1,2)$ (b)

\section{Distance Function in Continuous Space}

The optimization is carried out in $\mathbb{R}^{3}$ by finding the best shape of polyhedra corresponding to balls of constant radii using the proposed distance functions. To do this, the distance functions presented for the fcc and bcc grids in the previous section are stated in a more general form valid for all points $(x, y, z) \in \mathbb{R}^{3}$, where $x \geq y \geq z \geq 0$. The following distance functions are considered:

$$
\begin{aligned}
d_{\alpha, \beta}^{f c c}(\mathbf{0},(x, y, z) ; \gamma) & =\left\{\begin{array}{cc}
k \cdot \alpha & \text { if } x \leq y+z \\
(2 k-x) \cdot \alpha+(x-k) \cdot \beta & \text { otherwise, }
\end{array}\right. \\
\text { where } k & =\min _{k}: k \geq \max \left(\frac{x+y+z}{2}, x-(1-\gamma) k\right)
\end{aligned}
$$

and

$$
\begin{aligned}
d_{\alpha, \beta}^{b c c}(\mathbf{0},(x, y, z) ; \gamma) & =(2 k-x) \cdot \alpha+(x-k) \cdot \beta \\
\text { where } k & =\min _{k}: k \geq \max \left(\frac{x+y}{2}, x-(1-\gamma) k\right),
\end{aligned}
$$


where $k \in \mathbb{R}$ and $\gamma \in \mathbb{R}, 0 \leq \gamma \leq 1$ is the fraction of the steps where 2-steps are not allowed (so $\mathbf{1}_{B}^{k}$ and $\mathbf{2}_{B}^{k}$ corresponds to $\gamma k$ and $(1-\gamma) k$, respectively). In this way we obtain a generalization of the distance functions in discrete space $\mathbb{G}$ valid for all points $(x, y, z)$ where $x \geq y \geq z \geq 0$ in continuous space $\mathbb{R}^{3}$. By considering

$$
d_{\alpha, \beta}^{f c c}(\mathbf{0},(x, y, z) ; \gamma)=r \text { and } d_{\alpha, \beta}^{b c c}(\mathbf{0},(x, y, z) ; \gamma)=r
$$

the points on a sphere of constant radius are found.

Remark 1. For a fixed point $(x, y, z), \frac{x+y+z}{2}$ and $\frac{x+y}{2}$ are constant and $x-(1-\gamma) k$ is decreasing w.r.t. $k$ and when $k=0,0 \leq \max \left(\frac{x+y}{2}, x\right) \leq \max \left(\frac{x+y+z}{2}, x\right)$. Therefore both $k=\max \left(\frac{x+y+z}{2}, x-(1-\gamma) k\right)$ and $k=\max \left(\frac{x+y}{2}, x-(1-\gamma) k\right)$ has a solution $k \in \mathbb{R}$. Thus, when $k \in \mathbb{R}$,

$$
\begin{gathered}
k=\min _{k}: k \geq \max \left(\frac{x+y+z}{2}, x-(1-\gamma) k\right) \Leftrightarrow k=\max \left(\frac{x+y+z}{2}, x-(1-\gamma) k\right) \\
k=\min _{k}: k \geq \max \left(\frac{x+y}{2}, x-(1-\gamma) k\right) \Leftrightarrow k=\max \left(\frac{x+y}{2}, x-(1-\gamma) k\right)
\end{gathered}
$$

\subsection{Reformulation of $d^{f c c}$}

Using Remark 1, the expression for $d_{\alpha, \beta}^{f c c}$ is rewritten:

$$
\begin{aligned}
d_{\alpha, \beta}^{f c c}(\mathbf{0},(x, y, z) ; \gamma) & =\left\{\begin{array}{cc}
k \cdot \alpha & \text { if } x \leq y+z \\
(2 k-x) \cdot \alpha+(x-k) \cdot \beta & \text { otherwise, }
\end{array}\right. \\
\text { where } k & =\max \left(\frac{x+y+z}{2}, x-(1-\gamma) k\right) .
\end{aligned}
$$

We get two cases:

i) $\frac{x+y+z}{2} \geq x-(1-\gamma) k$

$$
\begin{aligned}
& k=\frac{x+y+z}{2} \Rightarrow \\
& \quad d_{\alpha, \beta}^{f c c}(\mathbf{0},(x, y, z) ; \gamma)=\left\{\begin{array}{cc}
\frac{x+y+z}{2} \cdot \alpha & \text { if } x \leq y+z \\
(y+z) \cdot \alpha+\left(\frac{x-(y+z)}{2}\right) \cdot \beta & \text { otherwise. }
\end{array}\right.
\end{aligned}
$$

ii)

$$
\begin{aligned}
& \frac{x+y+z}{2}<x-(1-\gamma) k \\
& k=x-(1-\gamma) k \Rightarrow k=\frac{x}{2-\gamma} \\
& d_{\alpha, \beta}^{f c c}(\mathbf{0},(x, y, z) ; \gamma)=\left\{\begin{array}{cc}
\frac{x}{2-\gamma} \cdot \alpha & \text { if } x \leq y+z \star \\
\left(2 \frac{x}{2-\gamma}-x\right) \cdot \alpha+\left(x-\frac{x}{2-\gamma}\right) \cdot \beta & \text { otherwise. }
\end{array}\right.
\end{aligned}
$$

Observe that when $x \leq y+z, \frac{x+y+z}{2} \geq x \geq x-(1-\gamma) k$ and thus the case * will not occur. 


\subsection{Reformulation of $d^{b c c}$}

Using Remark 1, the expression is rewritten also for $d_{\alpha, \beta}^{b c c}$ :

$$
\begin{aligned}
d_{\alpha, \beta}^{b c c}(\mathbf{0},(x, y, z) ; \gamma) & =(2 k-x) \cdot \alpha+(x-k) \cdot \beta \\
\text { where } k & =\max \left(\frac{x+y}{2}, x-(1-\gamma) k\right) .
\end{aligned}
$$

Again, there are two cases:

i) $\frac{x+y}{2} \geq x-(1-\gamma) k$

$$
k=\frac{x+y}{2} \Rightarrow
$$

$$
d_{\alpha, \beta}^{b c c}(\mathbf{0},(x, y, z) ; \gamma)=(y) \cdot \alpha+\left(\frac{x-y}{2}\right) \cdot \beta
$$

ii) $\frac{x+y}{2}<x-(1-\gamma) k$

$$
\begin{aligned}
k=x- & (1-\gamma) k \Rightarrow k=\frac{x}{2-\gamma} \\
& d_{\alpha, \beta}^{b c c}(\mathbf{0},(x, y, z) ; \gamma)=\left(2 \frac{x}{2-\gamma}-x\right) \cdot \alpha+\left(x-\frac{x}{2-\gamma}\right) \cdot \beta
\end{aligned}
$$

Together with (4), this describes the portions of polyhedra satisfying $x \geq y \geq$ $z \geq 0$. By using symmetry, the entire polyhedra are described. There polyhedra are spheres with the proposed distance functions.

\subsection{Optimization of the Parameters}

For any triplet $\alpha, \beta, \gamma(\alpha, \beta>0$ and $0 \leq \gamma \leq 1)$, (4) defines a polyhedron in $\mathbb{R}^{3}$. The shape of the polyhedra obtained for some values of $\alpha, \beta, \gamma$ are shown in Figure 3 Let $A$ be the surface area and $V$ the volume of the (region enclosed by the) polyhedron. The values of $A$ and $V$ are determined by the vertices of the polyhedra which are derived using (4) together with the expressions for $d_{\alpha, \beta}^{f c c}$ and $d_{\alpha, \beta}^{b c c}$ derived in Section 4.1 and 4.2, respectively. The vertices satisfying $x \geq y \geq z \geq 0$ are

$$
\begin{array}{r}
\left(\frac{2-\gamma}{\gamma \alpha+\beta-\beta \gamma}, \frac{\gamma}{\gamma \alpha+\beta-\beta \gamma}, 0\right) \text { and }\left(\frac{1}{\alpha}, \frac{1}{\alpha}, 0\right) \text { for } d^{f c c} \text { and } \\
\left(\frac{2-\gamma}{\gamma \alpha+\beta-\beta \gamma}, \frac{\gamma}{\gamma \alpha+\beta-\beta \gamma}, \frac{\gamma}{\gamma \alpha+\beta-\beta \gamma}\right) \text { and }\left(\frac{1}{\alpha}, \frac{1}{\alpha}, \frac{1}{\alpha}\right) \text { for } d^{b c c}
\end{array}
$$

The following error function (often called the compactness ratio) is used

$$
E=\frac{\frac{A^{3}}{V^{2}}-36 \pi}{36 \pi},
$$

which is equal to zero if and only if $A$ is the surface area and $V$ is the volume of a Euclidean ball.

The values of $\alpha, \beta$, and $\gamma$ that minimize $E$ are computed. The optimal values are found in Table 1 and visualized by the shape of the corresponding polyhedra in Figure 4. 

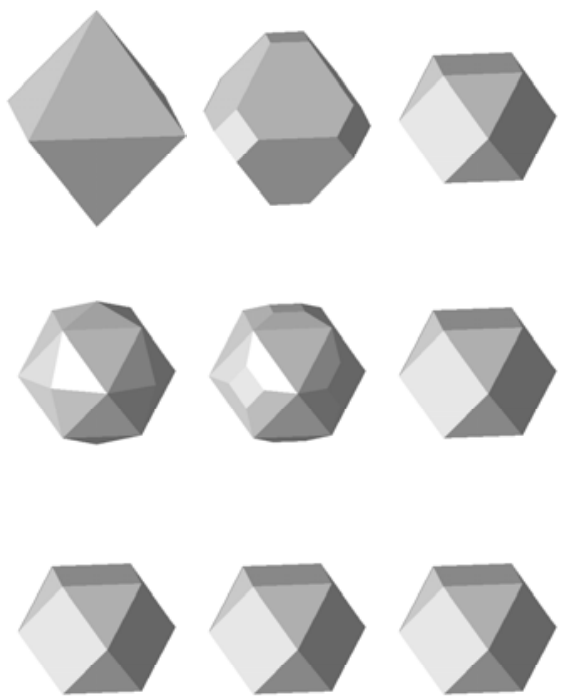
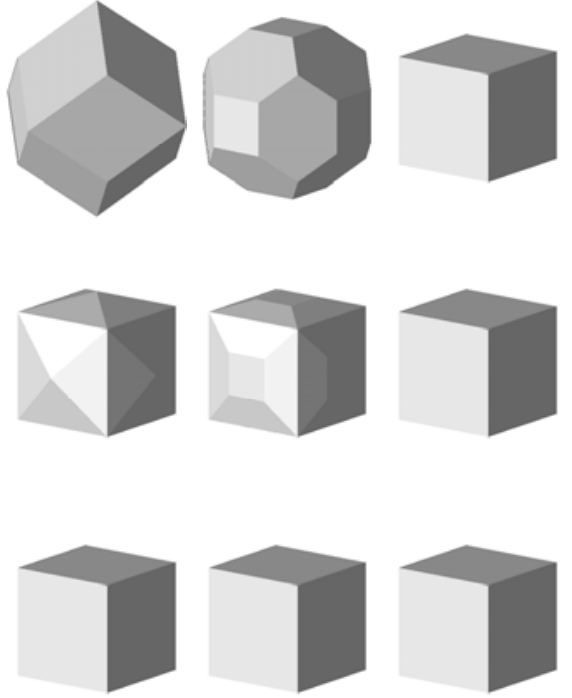

Fig. 3. Shapes of balls for $d_{\alpha, \beta}^{f c c}$ (left $3 \times 3$ set of figures) and $d_{\alpha, \beta}^{b c c}$ (right $3 \times 3$ set of figures) for $\alpha=1, r=1$ when $\beta=1$ (top row), $\beta=1.5$ (middle row), $\beta=2$ (bottom row) and $\gamma=0$ (left column), $\gamma=0.5$ (middle column), and $\gamma=1$ (right column) in each block

Table 1. Performance of weighted distance based on neighbourhood sequences (wns), weighted distance $(\mathrm{w})$, and distance based on neighbourhood sequences (ns) using the error function $E$. The value of $E$ is attained whenever $t$ is a strictly positive real number. The values shown in bold are fixed in the optimization.

\begin{tabular}{|l||l|l|l|l||l|l|l|l|}
\hline \multicolumn{1}{|c||}{} & \multicolumn{4}{c||}{ fcc } & \multicolumn{4}{c|}{ bcc } \\
\hline Name & $\alpha$ & $\beta$ & $\gamma$ & $E$ & $\alpha$ & $\beta$ & $\gamma$ & $E$ \\
\hline $\mathrm{w}$ & $t$ & $1.5302 t$ & $\mathbf{0}$ & 0.1367 & $t$ & $1.2808 t$ & $\mathbf{0}$ & 0.2808 \\
\hline $\mathrm{ns}$ & $\mathbf{1}$ & $\mathbf{1}$ & 0.8453 & 0.2794 & $\mathbf{1}$ & $\mathbf{1}$ & 0.5857 & 0.2147 \\
\hline $\mathrm{wns}$ & $t$ & $1.4862 t$ & 0.4868 & 0.1276 & $t$ & $1.2199 t$ & 0.4525 & 0.1578 \\
\hline
\end{tabular}

\section{Conclusions}

A new distance function is defined for the fcc and bcc grids, namely the weighted distance based on neighbourhood sequences. The value of the error function is lower for this distance function compared to the weighted distance and nsdistance. The weighted distances and ns-distances are special cases of the proposed distance function and the results in Figure 4 and Table 1 are similar (since another error function is used) to the weights obtained in 23] and equal to the neighbourhood sequences obtained in [4]. When $\gamma=0$ (the left columns in Figure 3), weighted distances are obtained and when $\alpha=1$ and $\beta=1$ (the top row in Figure 3), distances based on neighbourhood sequences are obtained. 


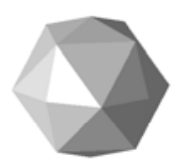

(a)

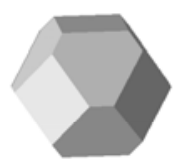

(b)

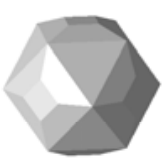

(c)

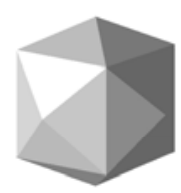

(d)

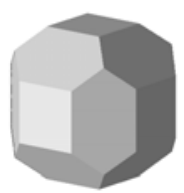

(e)

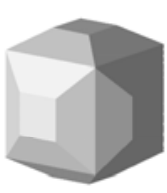

(f)

Fig. 4. Shapes of balls using values of $\alpha, \beta, \gamma$ that minimize $E$, see Table 1 (a) and (d): Weighted distance $(\gamma \equiv 0)$. (b) and (e): Distance based on neighbourhood sequences $(\alpha \equiv 1, \beta \equiv 1)$. (c) and (f): Proposed distance function. (a)-(c): fcc. (d)-(f): bcc.

For the balls in Figure 2, the neighbourhood sequence $B=(1,2)$ is used. This corresponds to $\gamma=0.5$, which approximates the optimal values quite good.

Since the distance function is path-based it will be a good choice in applications where a path-based distance function with low rotational dependency should be considered.

\section{References}

1. Matej, S., Lewitt, R.M.: Efficient 3D grids for image reconstruction using spherically-symmetric volume elements. IEEE Transactions on Nuclear Science 42(4), 1361-1370 (1995)

2. Strand, R., Borgefors, G.: Distance transforms for three-dimensional grids with non-cubic voxels. Computer Vision and Image Understanding 100(3), 294-311 (2005)

3. Fouard, C., Strand, R., Borgefors, G.: Weighted distance transforms generalized to modules and their computation on point lattices. Accepted for publication in Pattern Recognition. Available online, www.sciencedirect.com (2007)

4. Strand, R., Nagy, B.: Distances based on neighbourhood sequences in non-standard three-dimensional grids. Discrete Applied Mathematics 155(4), 548-557 (2006)

5. Carr, H., Theussl, T., Möller, T.: Isosurfaces on optimal regular samples. In: Bonneau, G.-P., Hahmann, S., C.D., H., (eds.) Proceedings of the symposium on Data visualisation 2003, Eurographics Association, pp. 39-48 (2003)

6. Coeurjolly, D., Miguet, S., Tougne, L.: 2D and 3D visibility in discrete geometry: an application to discrete geodesic paths. Pattern Recognition Letters 25(5), 561-570 (2004)

7. Verwer, B.J.H., Verbeek, P.W., Dekker, S.T.: An efficient uniform cost algorithm applied to distance transforms. IEEE Transactions on Pattern Analysis and Machine Intelligence 11(4), 425-429 (1989)

8. Borgefors, G.: Distance transformations in digital images. Computer Vision, Graphics, and Image Processing 34, 344-371 (1986)

9. Rosenfeld, A., Pfaltz, J.L.: Distance functions on digital pictures. Pattern Recognition 1, 33-61 (1968)

10. Rosenfeld, A., Pfaltz, J.L.: Sequential operations in digital picture processing. J. ACM 13(4), 471-494 (1966) 